\title{
Histochemical and Immunocytochemical Compartments of the Thalamic VPM Nucleus in Monkeys and Their Relationship to the Representational Map
}

\author{
E. Rausell and E. G. Jones \\ Department of Anatomy and Neurobiology, University of California, Irvine, California 92717
}

The ventral posteromedial nucleus (VPM) of the monkey thalamus was investigated with correlative anatomical and physiological techniques. On the basis of staining for cytochrome oxidase (CO), VPM is divided into a lightly stained, background matrix domain and an intensely stained rod domain. The latter consists of elongated rods of large, medium, and small cells, $500 \mu \mathrm{m}$ wide on average and extending anteroposteriorly, many of them through the full extent of the nucleus. The matrix, consisting of small cells, penetrates between the rods and expands at the dorsomedial, ventrolateral, and posterior aspects of VPM.

Multiunit mapping reveals that VPM contains a dorsally situated representation of the contralateral side of the head, face, eye, and interior of the mouth and a medially situated representation of the ipsilateral side of the lips and interior of the mouth, and that the same small region is represented in the same relative position through the full anteroposterior extent of the nucleus. Earlier work had shown that single CO rods contain the representation of the same portion of the periphery throughout their length. The present study suggests that rods in equivalent positions may represent the same portion of the periphery from animal to animal.

The cells of the rod and matrix domains show different patterns of immunoreactivity. Virtually all of the large- and medium-sized rod cells are immunoreactive for the calciumbinding protein parvalbumin, and many are stained by the monoclonal antibody CAT 301. Small GABA-immunoreactive cells and terminal-like puncta are highly concentrated in the rods but are dispersed in the matrix. In the matrix, all nonGABA cells are small, immunoreactive for 28-kDa calbindin, and not stained by CAT 301. They appear to form part of a wider system of calbindin-positive cells that extends into adjacent nuclei.

The $\mathrm{CO}$ rods are indicative of the modularity of the lemniscal component of the trigeminal part of the somatic sensory system at thalamic levels. Thalamocortical relay neurons in this compartment of VPM express a calcium-binding protein and a surface proteoglycan that distinguishes them from

\footnotetext{
Received June 12, 1990; revised Aug. 30, 1990; accepted Sept. 5, 1990.

This work was supported by Grants NS21377 and NS22317 from the National Institutes of Health, U.S. Public Health Service. E.R. was the recipient of a Fullbright Fellowship and a Fogarty International Fellowship. We thank Dr. P. C. Emson for the parvalbumin and calbindin antisera, Drs. C. Matute and P. Streit for the GABA monoclonal antibody, and Dr. S. Hockfield for the CAT 301 antibody.

Correspondence should be addressed to Dr. E. G. Jones, Department of Anatomy and Neurobiology, University of California, Irvine, CA 92717. Copyright (C) 1991 Society for Neuroscience $0270-6474 / 91 / 010210-16 \$ 03.00 / 0$
}

relay neurons in the matrix compartment of the nucleus. In the following paper (Rausell and Jones, 1991), the rod and matrix compartments are shown also to have different patterns of input and output connections.

Modularity is a predominant feature of the organization of the somatic sensory system. It is expressed at many different anatomical and physiological levels and was first recognized in the place- and modality-specific column of the somatic sensory cortex (Mountcastle, 1957; Powell and Mountcastle, 1959; Werner and Whitscl, 1968). At subcortical levels, particularly in the thalamus and dorsal column nuclei, modules represented by clusters of cells that are activated selectively by the same class of peripheral receptors appear to form independent and parallel channels for the flow of dorsal column-lemniscal information to the cortex and thalamus (Jones and Friedman, 1982; Dykes, 1983; Mountcastle, 1984; Florence et al., 1988).

Microelectrode mapping of the ventral posterior nucleus of the thalamus in Old World and New World monkeys has revealed that neurons over long anteroposterior traverses have closely similar receptive fields and modality properties (Dykes et al., 1981; Jones et al., 1982; Kaas et al., 1984). Mapping across the dorsoventral and mediolateral extents of the nucleus showed that these anteroposterior domains of cells with common place and modality properties have much shorter dorsoventral and mediolateral dimensions and can be construed as curved "rods" extending through the full anteroposterior extent of the nucleus (Jones and Friedman, 1982; Jones et al., 1982). In these earlier studies, it was also demonstrated that injections of horseradish peroxidase (HRP) made at regions of defined receptive field location in the first somatic sensory area (SI), and affecting $1 \mathrm{~mm}^{2}$ or less of cortex, retrogradely labeled ventral posterior neurons in similar, narrow-curving, anteroposteriorly extended rods (Jones et al., 1982). On this basis, it was proposed that functional rods in the ventral posterior nucleus projected to small columnlike domains in the SI cortex: the "rod-to-column" principle (Jones, 1985).

Subsequent to the above correlative physiological and anatomical studies, it was discovered in the ventral posterior medial subnucleus (VPM), containing the trigeminal representation, that staining for the mitochondrial enzyme cytochrome oxidase $(\mathrm{CO})$ provided a direct visualization of the place- and modality-specific rods of the thalamus. Dense concentrations of $\mathrm{CO}$ staining $250-500 \mu \mathrm{m}$ in width in frontal sections extend anteroposteriorly through the full anteroposterior extent of VPM (Jones et al., 1986a). These are coextensive with similar rodlike concentrations of neurons immunoreactive for $\gamma$-aminobutyric acid (GABA), and microelectrode mapping in these animals revealed 
that a given $\mathrm{CO}$ rod represents the same set of receptive field locations throughout its full anteroposterior extent (Jones et al., 1986b). This and the earlier retrograde labeling in VPM after small injections of parts of the face representation in SI would tend to support the concept that the $\mathrm{CO}$ rods are the same as the functionally defined rods.

The purpose of the present study is to provide a more comprehensive picture of the $\mathrm{CO}$ rod pattern and its relationship to the functional map in VPM, and to show that the rods and the matrix in which they lie are distinguished by the presence of thalamic neurons that are characterized by the expression of different calcium-binding proteins (Jones and Hendry, 1989).

A preliminary report has appeared (Rausell and Jones, 1989).

\section{Materials and Methods}

The material is drawn from the brains of 32 adult cynomolgous monkeys (Macaca fascicularis) weighing 3.0-3.5 kg. Twelve animals were used in new experiments. Twenty animals formed part of previous physiological (Jones et al., 1982, 1986a,b) or immunocytochemical studies (Hendry et al., 1988; Jones and Hendry, 1989). The new experiments included 10 normal monkeys, which were used for immunocytochemistry, and 2 used for additional microelectrode mapping. The material from the earlier studies provided data relevant to the construction of physiological maps and included additional material stained by the monoclonal antibody CAT 301 and stained for CO, GABA, or the calcium-binding proteins.

Immunocytochemical studies. Animals were deeply anesthetized with intravenous Nembutal and perfused through the left ventricle with 300 $\mathrm{ml}$ saline, followed by 3 liters of a fixative solution containing $2.0 \%$ or $4.0 \%$ paraformaldehyde and $0.1 \%$ or $0.2 \%$ glutaraldehyde in $0.1 \mathrm{M}$ phosphate buffer ( $\mathrm{pH}, 7.4)$, then successively with $10 \%, 15 \%$, and $20 \%$ sucrose in phosphate buffer. The brains were removed and infiltrated with $30 \%$ sucrose in phosphate buffer. They were then blocked and frozen in dry ice. The thalami were serially sectioned in the frontal, sagittal, or horizontal plane on a sliding microtome into 6 series of alternating $15-\mu \mathrm{m}$ and $30-\mu \mathrm{m}$ sections. Every $15-\mu \mathrm{m}$ section was stained, free floating, for immunocytochemistry. The localization of GABA was detected by means of either a mouse monoclonal antibody (Matute and Streit, 1986) or a commercial rabbit anti-GABA antiserum (Chemicon). Parvalbumin was localized by means of a sheep antiparvalbumin antiserum and calbindin by a rabbit anti-calbindin antiserum (see Jones and Hendry, 1989). A mouse monoclonal antibody, CAT 301, made against aldehyde-fixed cat spinal cord (McKay and Hockfield, 1982; Hockfield and McKay, 1983; Hendry et al., 1984) was used to stain certain previously characterized populations of cells (Hendry et al., 1988). The staining protocol with CAT 301 was the same as used in these earlier studies. The other monoclonal antibodies and antisera used were diluted in $100 \mathrm{~mm}$ phosphate buffer $(\mathrm{pH}, 7.4)$ containing $0.25 \%$ Triton $\mathrm{X}-100$ and $3 \%$ normal serum of the species in which the secondary antibody was raised. Final dilutions of the antibodies were 1:3000 for anti-parvalbumin, anti-calbindin, and anti-GABA antiserum and 1:7500 for anti-GABA monoclonal antibudy. The sections processed immunocytochemically for the detection of a single substance were preincubated for $1-2 \mathrm{hr}$ in phosphate buffer containing 3\% normal serum and $0.25 \%$ Triton at $4^{\circ} \mathrm{C}$, then incubated in a solution of the primary antibody in phosphate buffer, 3\% normal serum, and $0.25 \%$ Triton for $24-36 \mathrm{hr}$ at $4^{\circ} \mathrm{C}$. The sections were then washed thoroughly and stained by the avidin-biotin-peroxidase method ( $\mathrm{ABC}$, Vectastain). Immunoreactivity was finally developed by incubation in a solution of $0.02 \% 3,3^{\prime}$-diaminobenzidine tetrahydrochloride plus $0.001 \%$ hydrogen peroxide. The alternating series of $30-\mu \mathrm{m}$ sections was stained histochemically for cytochrome oxidase (CO; Wong-Riley, 1979), for acetylcholinesterase (AChE; Woolf and Butcher, 1981), or stained with thionin or cresyl violet. Controls for the immunocytochemical staining consisted of omitting the primary antisera from the staining procedure, preadsorption of the anti-GABA antibodies by a GABA-albumin conjugate, and preadsorption of the anti-parvalbumin or anti-calbindin antisera with 10-15 $\mu \mathrm{g}$ per $\mathrm{ml}$ parvalbumin or calbindin (Sigma).

The sections were studied under transmitted light microscopy, using bright- and dark-field illumination. Camera lucida drawings were made at a magnification of $20 \times$ of the VPM nucleus in adjacent thionin-, immunocytochemically, and CO-stained sections. Cut profiles of blood vessels were drawn on each, and in the CO- and parvalbumin-stained sections, the outlines of the major densities of staining were drawn, usually under dark-field illumination. The thionin-stained sections were used to delimit the nucleus and the major concentrations of small and large cells in it. The drawings were then superimposed, using the blood vessel profiles as guides. Serial photomicrographs of frontal sections through the anteroposterior extent of the $\mathrm{CO}$ - and parvalbumin-stained VPM were printed at high contrast and photocopied, and the outlines of the densest concentrations of stain were traced sequentially from section to section. Selected CO-stained sections were digitized and analyzed in an MCID (Imaging Devices Inc.) image analysis system in order to show the CO-stained densities.

Counts of cells were made directly from camera lucida drawings at high magnification on which all cells were plotted in relation to the outlines of the CO-stained densities, or by direct microscopic observation in relation to an eyepiece reticule that, at $40 \times$, defined an area approximately $250 \times 250 \mu \mathrm{m}$. Proportions of cells were determined by counting an identical area in the adjacent thionin-stained section.

Multiunit mapping. The left and right VPM nuclei of 2 Macaca fascicularis monkeys additional to the original 10 reported in Jones et al. $(1982,1986 \mathrm{~b})$ were mapped by means of microelectrodes. Under intravenous Nembutal anesthesia, epoxy-insulated, stainless-steel microelectrodes (impedance 5-15 $\mathrm{m} \Omega$ ) were introduced vertically into the VPM through the overlying cerebral cortex. VPM was then mapped systematically in a stereotaxic grid pattern, electrodes being inserted at $200-400-\mu \mathrm{m}$ intervals. Single and multiunit responses to gentle stimulation with hand-hcld probes of the skin of the head and face and of the mucous surfaces of the interior of the mouth were systematically recorded in $50-100-\mu \mathrm{m}$ steps. Small electrolytic marking lesions were made at recorded depths on selected tracks for purposes of subsequent identification.

At the conclusion of the experiment, the animals were deeply anesthetized with intravenous Nembutal and perfused with $4 \%$ paraformaldehyde and $0.2-0.5 \%$ glutaraldehyde in $0.1 \mathrm{~m}$ phosphate buffer. The brains were removed, infiltrated with sucrose, and frozen, and the thalami were serially sectioned at $50 \mu \mathrm{m}$ in the frontal plane. Alternate sections were stained for $\mathrm{CO}$ or with thionin. Camera lucida drawings were made of the VPM nucleus, and the contained CO-stained rods and electrode tracks and recording sites were reconstructed on them as indicated above, and by correlation with the positions of lesions. They were then correlated with the reccptive field data recorded at the time of the experiment. It should be noted that the introduction of multiple closely spaced electrodes damages large parts of the nucleus, and the tracks and associated damage obscure the CO-staining pattern seen in normal animals. Similarly, accumulating damage makes it rarely feasible to map the entire nucleus of single animals at such closely spaced intervals. Hence, a map correlated with the $\mathrm{CO}$ pattern and a complete map of the nucleus can only be built up from several partial maps.

\section{Results}

Nissl and cytochrome oxidase staining

VPM of the monkey, as defined, for example, by Olszewski (1952), is an L-shaped structure in frontal sections with the stem of the $\mathrm{L}$ oriented vertically and the foot of the $\mathrm{L}$ directed medially. The neuronal population in Nissl-stained material is inhomogeneous. Large and medium ( $20-40 \mu \mathrm{m}$ in diameter), darkly stained angular cells are arranged in irregular clumps $200-800$ $\mu \mathrm{m}$ in diameter that are not clearly delimited and tend to merge with one another at intervals. When separate, the intervening regions form thin, irregular septa, made up of small cell somata (10-20 $\mu \mathrm{m}$ in diameter), and commonly oriented approximately mediolaterally across the nucleus (Fig. 1). The clumps of large cells also contain smaller cells, $10-20 \mu \mathrm{m}$ in diameter, many of which are clearly neurons. Larger regions containing only small cells are also found along the dorsomedial edge of VPM, wherc it abuts the lamina separating it from the centre médian (CM) or anterior pulvinar (Pla) nuclei, and at the ventrolateral aspect of VPM, where it abuts on the ventral posterior inferior (VPI) and ventral medial basal (VMb) nuclei (Fig. 1). 

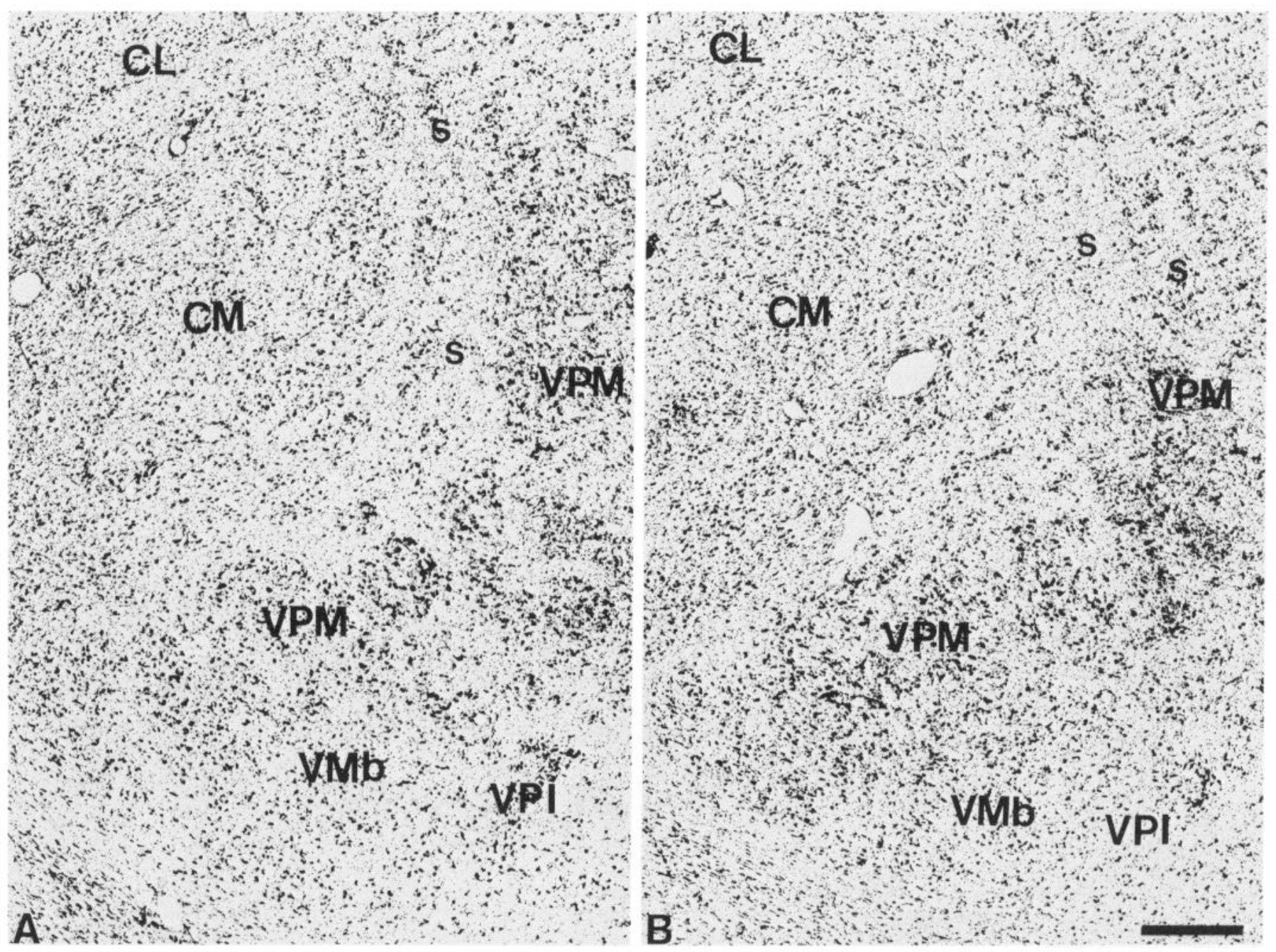

Figure 1. Nissl-stained frontal sections through $V P M$ and adjacent nuclei showing the small-celled $(s)$ regions of the nucleus, especially seen where they abut on the capsule of the $C M$. Scale bar, $500 \mu \mathrm{m}$.

The CO-staining pattern is also not homogeneous in VPM. Two main CO-positive domains can be distinguished, especially in $\mathrm{CO}$ material that is not overstained. The principal domain (the rod domain) is composed of densely CO-stained clumps $100-800 \mu \mathrm{m}$ in diameter, which, serial section analysis shows, are continuous from section to section and thus form parts of anteroposteriorly oriented rodlike structures (Figs. 2-4). The rods occupy both parts of the $\mathrm{L}$ and correlate very closely with the clusters of large cells that can be seen in the Nissl-stained sections, except that the outlines are clearer in the $\mathrm{CO}$ material, especially when analyzed in high-contrast photographs or digitized (Fig. 5). The matrix in which these $\mathrm{CO}$ rods are embedded represents the second domain (the matrix domain) and characteristically shows a somewhat weaker, homogeneous, and unclumped CO-staining pattern. It is the presence of this weakerstained matrix that gives the rods their outlines. $\mathrm{CO}$ rods have their clearest borders and are most consistent in size and position from section to section in the medial, horizontal division of the nucleus and over the middle part of the anteroposterior extent of the nucleus (Fig. 3). Anteriorly and posteriorly, they tend to merge with one another. In the vertical division, the major densities of $\mathrm{CO}$ staining, though traceable from section to section, can show less contrast between rod and matrix in some sections, and thus, their exact outlines are hard to deter- mine, and they tend to merge with their neighbors. Sagittal sections (Fig. 6) show the continuity quite well, though the curved nature of the rods prevents their complete demonstration in a single section. The sagittal sections show merging of some rods (Fig. $5 B$ ) and suggest that each may contain subsidiary concentrations of $\mathrm{CO}$ staining. Certain rods are broken up by the passage of fiber bundles through them (Figs. 2, 3, 6).

The matrix intervenes between the rods and surrounds both them and the marginal rim of the nucleus, forming an enclosing envelope to the latter. The envelope is thin at the anterior pole of VPM, but the area of detectable matrix enlarges as it extends into middle and posterior levels of the nucleus. At these levels, the envelope is particularly thick dorsomedially, in the medial concavity of the $\mathrm{L}$, where it borders the anterior pole and ventral margin of the Pla, and ventrolaterally, where VPM abuts on VPI and VMb. VPI and VMb are themselves stained homogeneously and with a density very similar to that of the VPM matrix. The borders of Pla can be more easily demarcated because this nucleus shows slightly stronger $\mathrm{CO}$ staining than the VPM envelope (Figs. $2 B, 5 B$ ).

Superimposition of thionin- and CO-stained sections, of the type illustrated in Figures 1 and 2, shows that the $2 \mathrm{CO}$ domains in VPM correspond to the 2 zones of Nissl-stained elements. The rods correspond to the clusters of large, medium, and small 

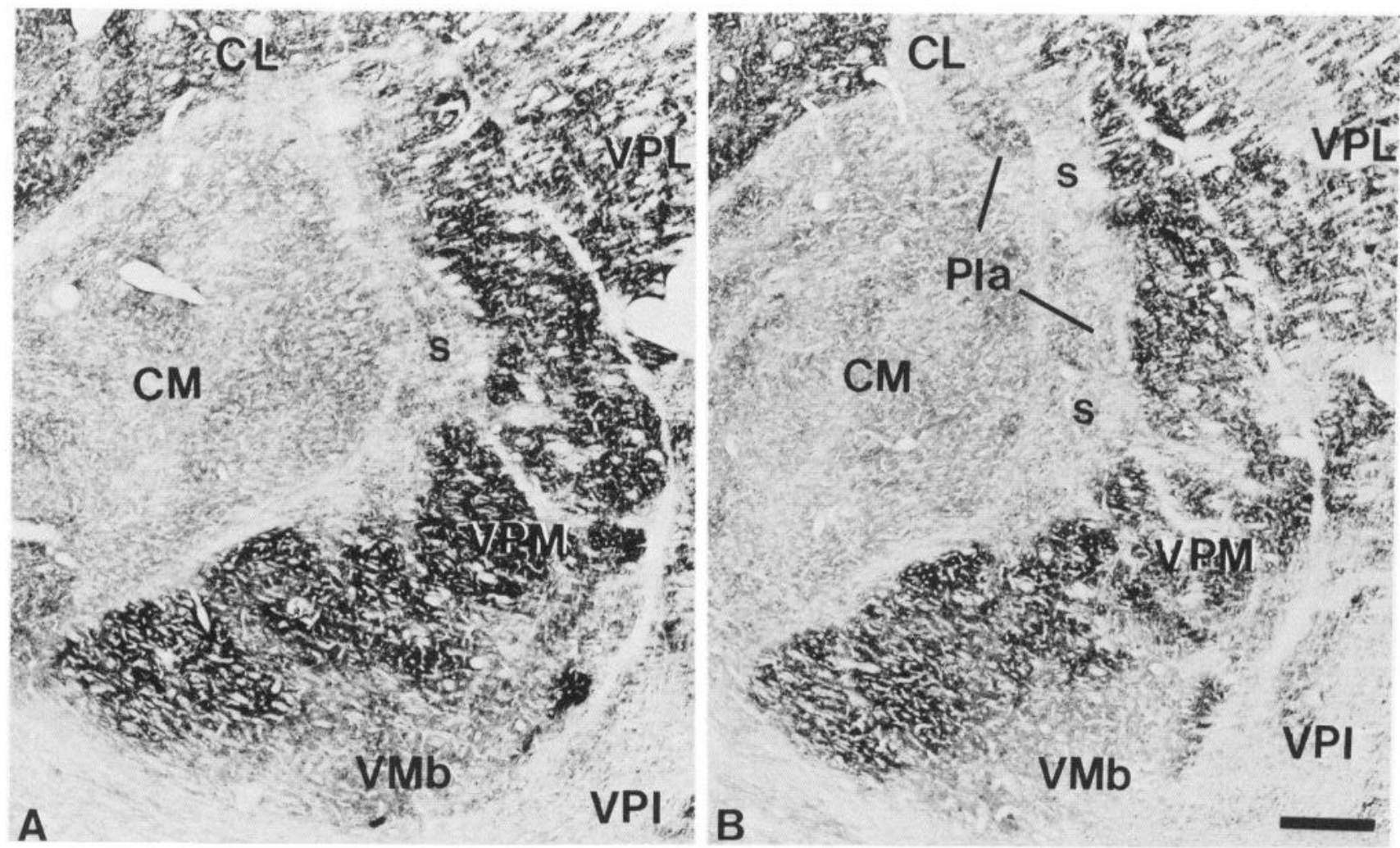

Figure 2. CO-stained frontal sections. $A$ is closely adjacent to the Nissl-stained section in Figure $1 A$. $B$ is immediately posterior to that shown in Figure $1 B$. These show the CO-dense rods, intervening weaker matrix, and the enlargement of the latter at $s$. In $B$, note how the anterior pole of the Pla nucleus (with slightly denser CO stain) intrudes on the small-celled matrix (s) region. Scale bar, $500 \mu$ m. $V P L$, ventral posterior lateral nucleus.

cells, whereas the matrix corresponds to regions in which neurons are small and sparsely distributed. The sizes and staining character of cells in the VPM matrix are quite similar to those of cells in LV, PI, VMb, and Pla, but in the matrix, the cells are less densely packed than in the 3 adjoining nuclei.

\section{The representation pattern in VPM}

Figure 7 shows the representation derived from single and multiunit mapping of VPM in 1 of the 2 newly mapped animals. Each receptive field has been correlated with the positions of single and multiunit recording sites on the basis of lesion position and stereotaxic coordinates recorded at the time of the experiment and has been added to camera lucida tracings of the outlines of VPM sections at known anteroposterior levels from the animal mapped. The damage caused by multiple closely spaced electrode penetrations prevents adequate delineation of $\mathrm{CO}$ rods in experiments of this type. Increasing damage as recording proceeds similarly prevents complete mapping of VPM at such close spacing in any animal. The map in this animal is therefore incomplete medially and laterally. Figure 8 presents a combined map in which the microelectrode maps derived from the 10 animals have been consolidated. This consolidation was done by plotting the positions of units or multiunit clusters with receptive fields on the same, larger body regions indicated, against stereotaxic position in relation to the borders of each nucleus examined. These were then matched to the borders of the single normal nucleus. It is evident that the greater part of the medial, horizontal part of VPM (the foot of the $\mathrm{L}$ ) is devoted to the representation of ipsilateral intraoral structures, almost exclusively (except for the ipsilateral hard palate) those innervated by the mandibular division of the trigeminal nerve. The ipsilateral representation is at its widest in the middle part of the nucleus (AP +6.5 to $\mathrm{AP}+5)$ and is smaller at the anterior and posterior poles. The vertical part of VPM contains an extensive representation of the contralateral field of innervation of the trigeminal nerve, including its ophthalmic, maxillary, and mandibular divisions. The ophthalmic representation is the smallest and occupies the dorsalmost part of VPM that has the shortest anteroposterior extent. The mandibular representation abuts upon the ipsilateral representation. No region was identified in which single units or multiunit clusters have bilateral receptive fields.

Reconstruction of electrode tracks with lesion sites in this and the previous studies suggest that the majority of responding units and multiunit clusters in the mapping experiments were recorded from neurons in the $\mathrm{CO}$ rod domain. Lesions indicating recording sites commonly intruded, however, on the matrix domain between individual rods. Because the matrix regions are very thin and because of the low resolving power of the microelectrodes used here (not better than $100 \mu \mathrm{m}$; Jones and Friedman, 1982), the method is insufficient to distinguish cells at the edges of rods from those of the intervening and surrounding matrix. It is therefore possible that the map would also include responding neurons in the matrix regions. When electrodes traversed the somewhat thicker, CO-weak matrix region in the posteromedial concavity of VPM, units were not driven by the types of stimuli applied in this study. 

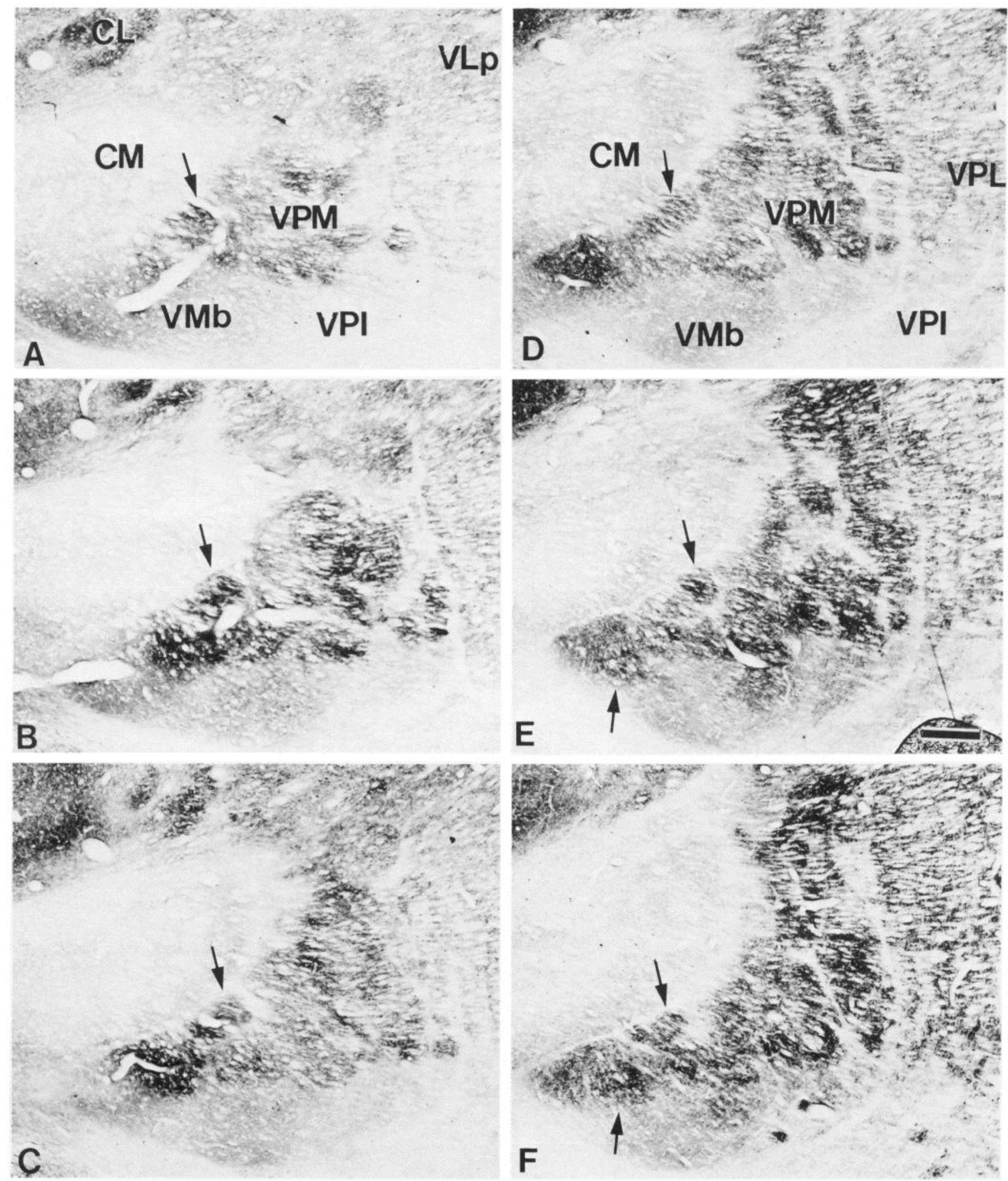

Figure 3. CO-stained frontal sections in anteroposterior order $(A-L)$ and at approximately 200 - $\mu \mathrm{m}$ intervals, showing CO-stained rods over their full extent (from a monkey different from that shown in Figures 1 and 2). In $A-I$, one of the upper rods is indicated by arrows. This merges posteriorly with its neighbors in $J$. In $E-L$, a different lower rod is arrowed. In $E$, it merges anteriorly with another rod. Scale bars, $500 \mu$ m. 


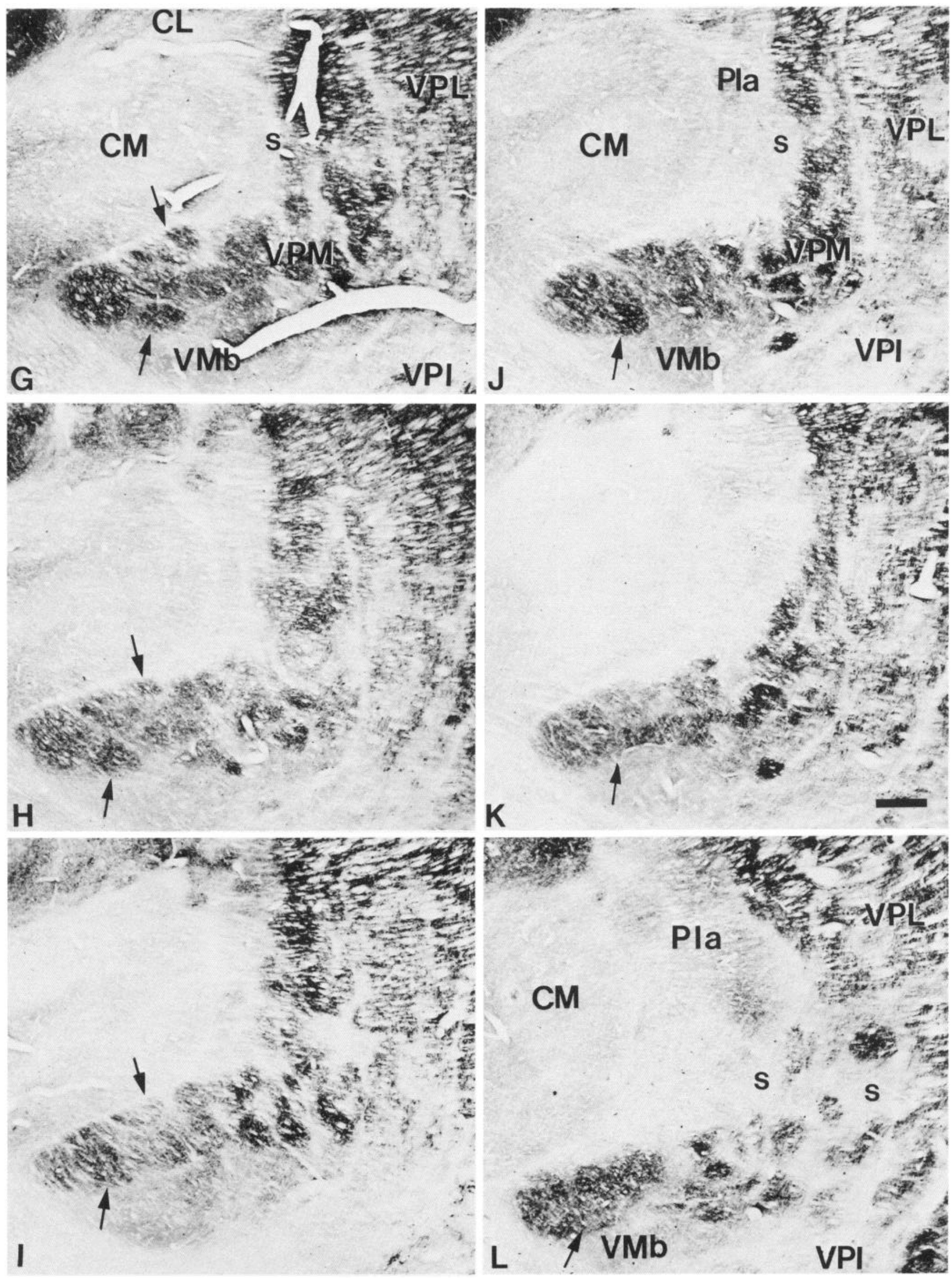


Figure 4. Tracings made from highcontrast photocopies of photomicrographs shown in Figure 3, to illustrate the mode of outlining CO-stained rods. $s$ indicates the CO-weak matrix region. Asterisks and arrows indicate $2 \mathrm{CO}$ stained rods that can be followed throughout the series.

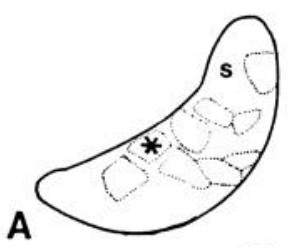

A

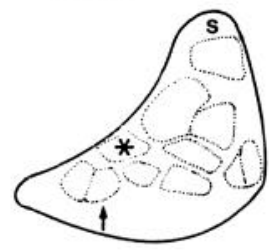

B

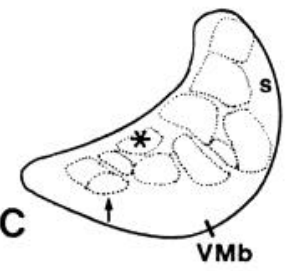

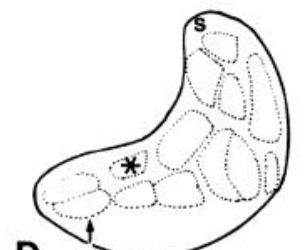

D

E

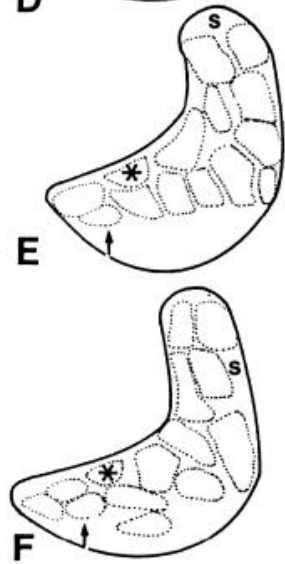

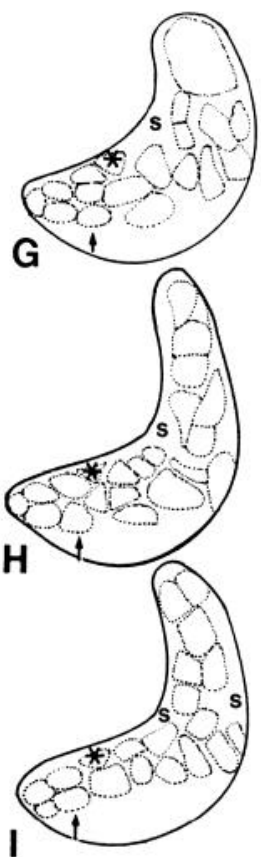

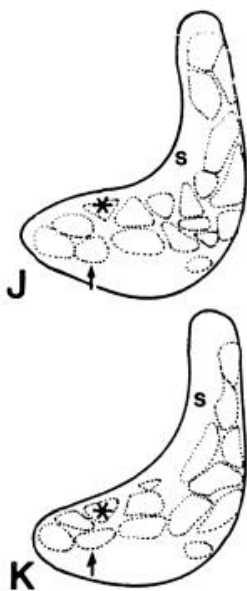

L

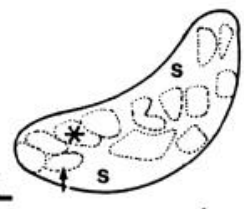

\section{Immunocytochemistry}

GABA-, CAT 301-, or parvalbumin-immunoreactive staining in VPM always appears in a rodlike pattern very comparable to that seen with CO staining (Figs. 9, 12). At low magnification and after superimposing photographic images or camera lucida outlines of the rods stained for $\mathrm{CO}$ in one section with those stained by immunocytochemistry in adjacent sections, it becomes very apparent that GABA rods, CAT 301 rods, and parvalbumin rods (Figs. 9, 11, 12) coincide with one another and with the rods of $\mathrm{CO}$ staining. By contrast, calbindin-immunoreactive staining in VPM (Figs. 10,11) is always in a pattern complementary to the rodlike pattern and coincides with the matrix $\mathrm{CO}$ domain.

\section{Immunostaining for calcium-binding proteins}

Parvalbumin-positive cells in VPM are confined to the rods (Figs. 9, 11). They have large- and medium-sized somata (25$40 \mu \mathrm{m}$ in diameter), as illustrated in the following paper (Rausell and Jones, 1991). The rods also show dense concentrations of parvalbumin-positive fibers, and parvalbumin-positive fibers pass between the rods in large numbers. Parvalbumin-positive cells tend to be arranged in one or more small groups of 20-30 neurons within the rods. In most rods, counts over defined areas
Figure 5. Digitized images of 2 COstained sections from third monkey showing focal concentrations of stain in each rod. Scale bar, $500 \mu \mathrm{m}$.
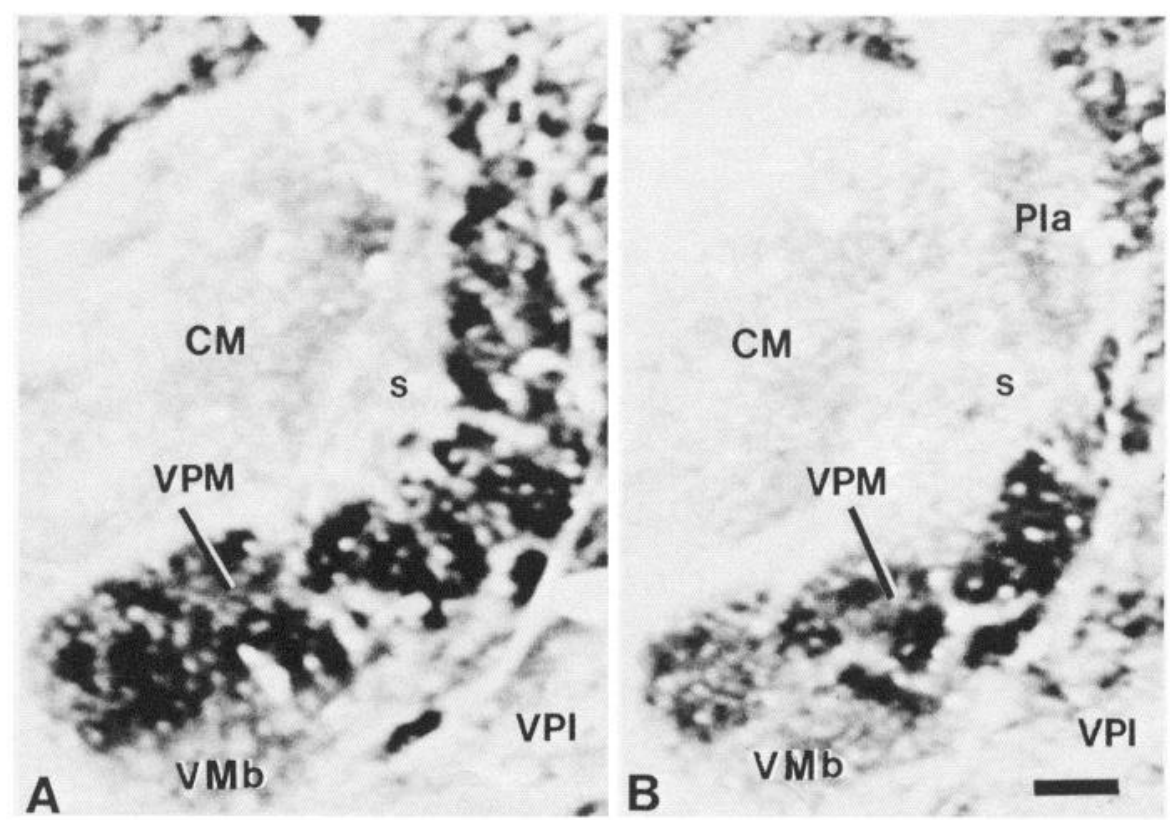

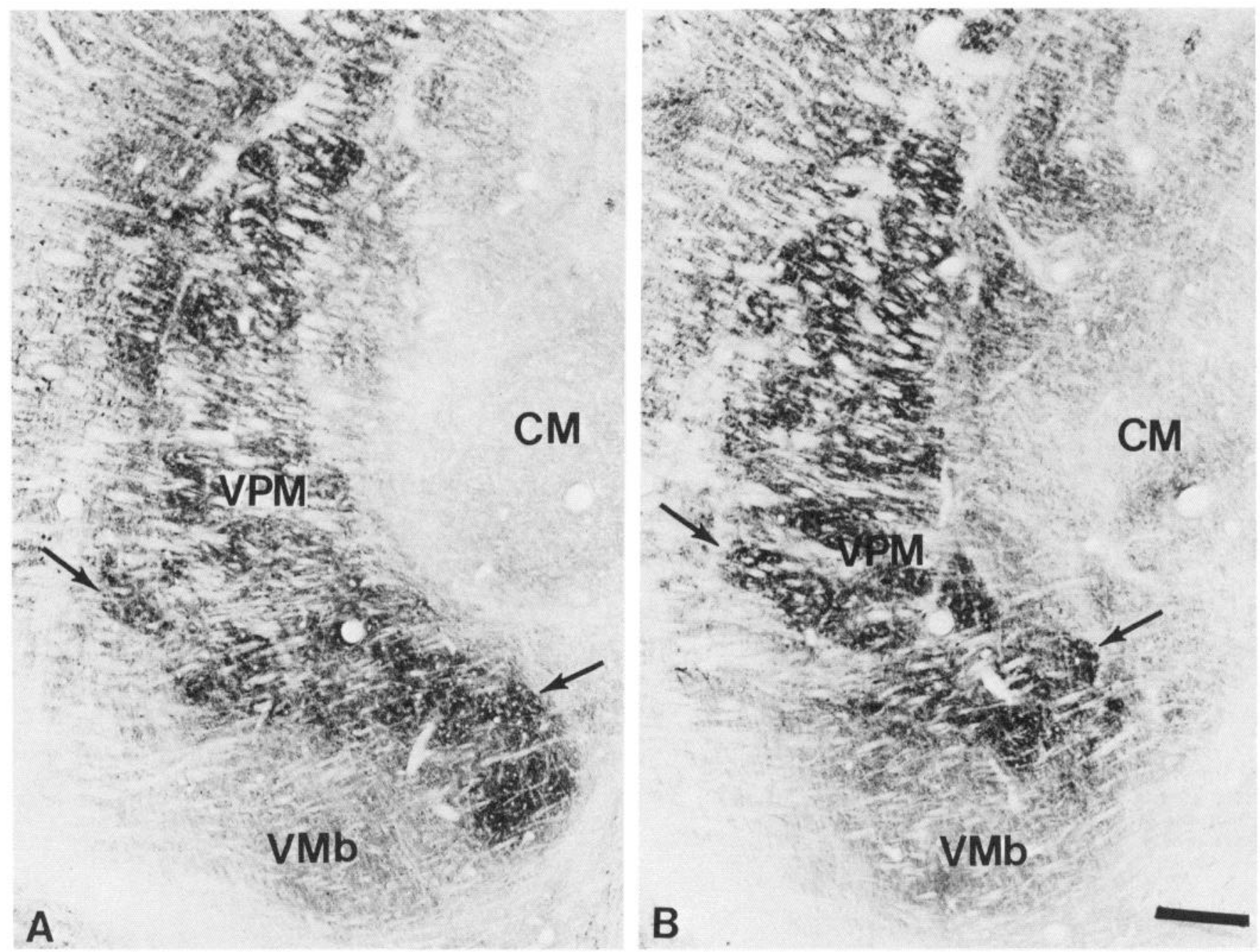

Figure 6. CO-stained sagittal sections from a series and approximately $250 \mu \mathrm{m}$ apart (note same circular blood vessel profiles in $V P M$ and $C M$ ). Anterior is to the left. Arrows indicate portions of the same curved rod, which shows focal concentrations of stain and appears to bifurcate in $B$. Scale bar, $500 \mu \mathrm{m}$.

(see Materials and Methods) show that they make up $80-100 \%$ of the large- and medium-cell population. There appear to be fewer in the most medial 2 rods, whose staining is reduced in comparison with other rods, especially posteriorly in the nucleus. Few or no parvalbumin-positive cells can be seen within the matrix $\mathrm{CO}$ domain, though parvalbumin-positive fibers pass through it from the trigeminal lemniscus; the stained fibers of the lemniscus clearly enter VPM by passing through VPI in large numbers (Figs. 9, 11). VMb is virtually free of immunoreactivity for parvalbumin. No immunoreactive cells are found in it, and any stained fibers appear to be passing through it towards VPM. Pla is also free of cell and fiber staining for parvalbumin, but CM shows intense staining of large numbers of cells and a densely immunoreactive neuropil (Figs. 9, 11).

Calbindin-positive somata in VPM (Figs. 10, 11), are distributed in a pattern complementary to that of parvalbuminstained somata. They are small in size (15-20 $\mu \mathrm{m}$ in diameter; see Fig. 13 and Rausell and Jones, 1991). Most calbindin-positive cells are found in a region almost exactly corresponding to the CO matrix region (Figs. 10,11). Like the latter, it extends from the anterior pole of VPM, where it is very thin, to the dorsomedial enlargement, where it borders the emerging ante- rior pole of Pla. Some calbindin-positive cells intrude into the rods, especially the most medial 2 rods, which, unlike others, contain a relatively dense concentration of calbindin-positive fibers (Fig. 13B). No special suborganization can be observed in the calbindin-positive population of cells, though medially, along the border with $\mathrm{Pla}$ and $\mathrm{CM}$, they tend to aggregate in small and irregular clusters (e.g., Fig. $11 C$ ). $\mathrm{VMb}$ and VPI contain numerous calbindin-immunoreactive cells and fibers. The stained cell populations of both nuclei are continuous, without interruption, with that in the matrix domain of VPM. Pla shows dense cell and fiber immunoreactivity for calbindin, while CM contains a few calbindin-positive cells but is largely free of calbindin immunoreactivity in the neuropil. The borders of Pla are clearly demarcated from VPM, because the former has a greater packing density of calbindin-positive cells.

\section{GABA immunostaining}

GABA immunoreactivity is displayed by small neuronal somata $10-15 \mu \mathrm{m}$ in diameter, and by their processes in VPM. Both the rod and matrix $\mathrm{CO}$ domains contain GABA-positive cells, 
Figure 7. Outlines of 3 frontal sections $($ left $)$ at approximately $500-\mu \mathrm{m}$ intervals showing reconstructions of electrode tracks and marker lesions (dots) from part of an experiment in which VPM was mapped at close-spaced intervals. Cross bars and numbers indicate depths over which units and multiunit clusters having receptive fields, fied. Note how a single region tends to sition through the full anteroposterior extent of the nucleus. as indicated on the right, were identibe represented at the same relative po-
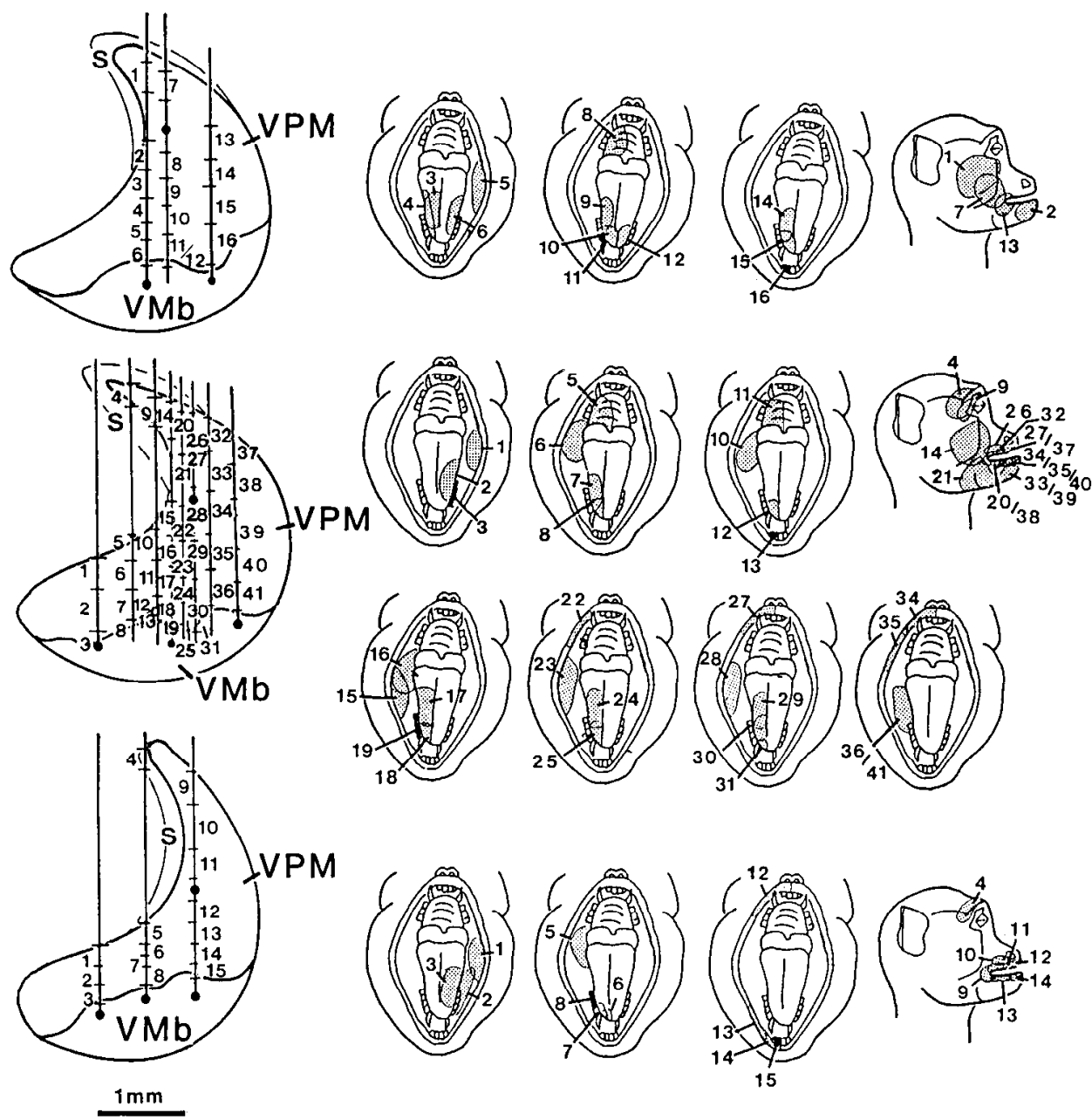

but the rod domain contains a much denser concentration of GABA-positive processes and punctate, terminal-like structures (Figs. 12-14). These occupy the entire extent of each rod and are responsible for its denser-staining appearance at low magnification (Fig. 11B). GABA neurons are unevenly distributed throughout the interior of the rod; they tend to concentrate in small clumps within the rod (Fig. 12; see Rausell and Jones, 1991, their Fig. 6), but with no obvious consistency in regard to location from rod to rod. In the matrix $\mathrm{CO}$ domain, GABA neurons are more sparsely distributed, stained processes and puncta are less densely concentrated, and the cells are not arranged in any obvious pattern (Fig. $13 A, B$ ).

\section{CAT 301 immunostaining}

CAT 301 staining also forms a rodlike pattern (Fig. 12A), and staining can be observed in neuronal somata, processes, and fibers. CAT 301-positive cells are large and medium sized (25$40 \mu \mathrm{m}$ in diameter) and tend to appear in small groups confined to variable sectors of rods. CAT 301 staining could be found in 25-45 neurons within rod areas 100-800 $\mu \mathrm{m}$ wide in a single section. The number corresponds to approximately 35$55 \%$ of the Nissl-stained neurons counted in the same area of adjacent sections. Differences in the number and staining intensity of CAT 301-positive neurons and fibers can be observed between the ipsilateral and the contralateral representations of
VPM. The neuropil in rods of the ipsilateral region is less intensely stained by CAT 301, and there is also a slight decrease in the number of stained neurons in comparison with the rods of the contralateral representation (Fig. 12A). A comparison of alternate sections stained separately for GABA and by CAT 301 reveals that, in a single rod, clumps of CAT 301-positive cells and clumps of GABA cells do not overlap, though occasional GABA-and CAT 301-positive cells can intermingle (not illustrated). Previous studies have shown that no cells in VPM co-stain for GABA and CAT 301 (Hendry et al., 1988). No CAT 301-positive cells can be seen in the matrix CO domain (Fig. 12A). The adjacent Pla, CM, and VPI nuclei are devoid of CAT 301 staining. VMb shows faint neuropil staining, without cell somal staining, especially adjacent to the medial tip of VPM.

\section{Discussion}

The present results indicate that the VPM nucleus of the monkey thalamus is divided into compartments that can be distinguished histochemically and immunocytochemically. The following paper (Rausell and Jones, 1991) shows that these compartments also have different input-output relationships. One of the compartments, the rod domain, is clearly modular in organization, and the present studies, taken in conjunction with 


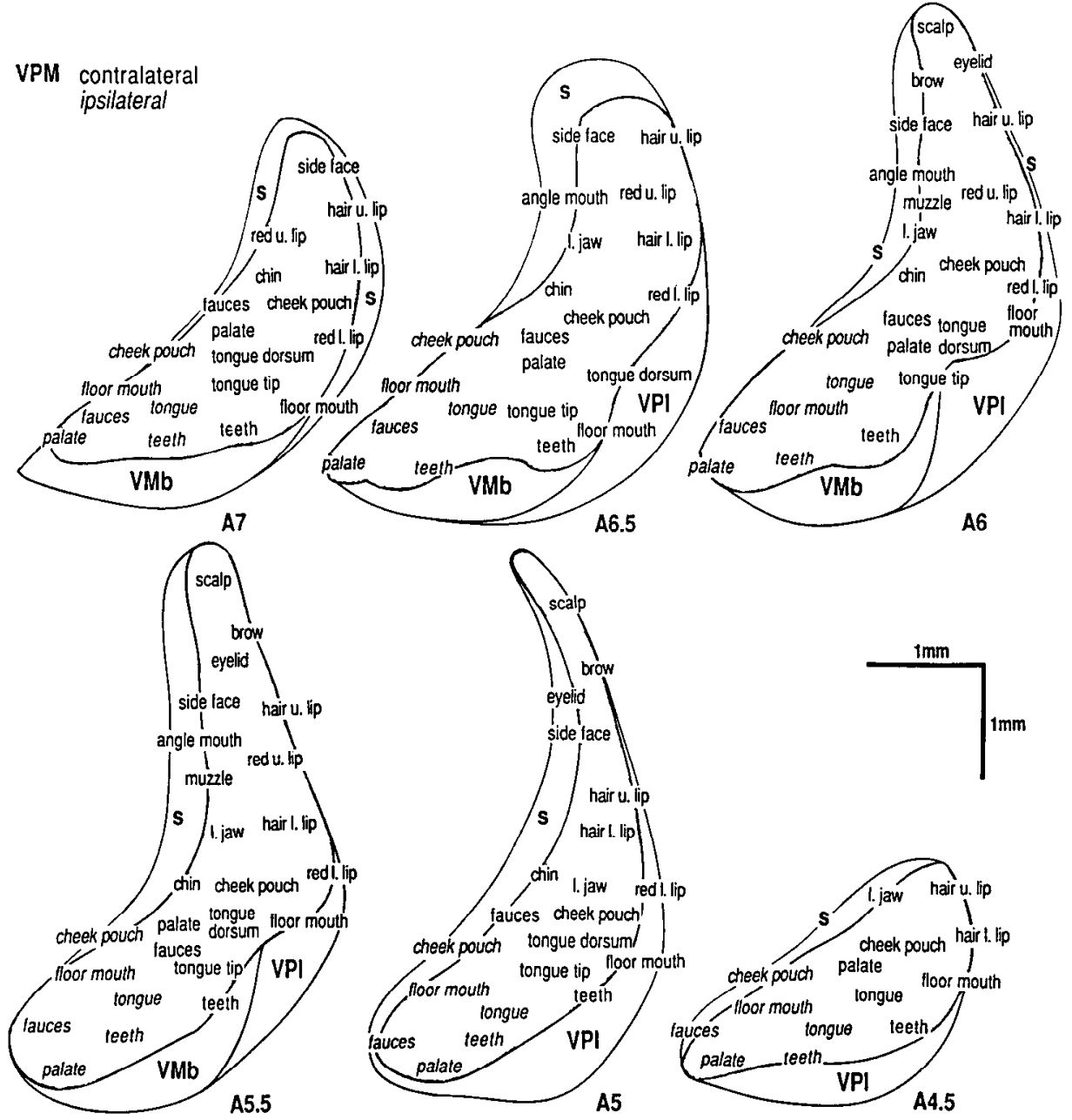

Figure 8. Camera lucida drawings from a single brain, with stereotaxic coordinates derived from measurements made during electrode penetrations (bottom right of each drawing). $s$ indicates the CO-weak, small-celled domain. Ipsilateral (italic type) and contralateral (roman type) representations obtained from consolidation of the results from multiunit mapping in 10 animals are shown. For further details, see Results. our earlier results, suggest that it forms the route through which place- and modality-specific information of the classical lemniscal type is relayed to the SI area of the cerebral cortex. The second compartment, the matrix domain, appears more diffuse in its organization, and the following paper (Rausell and Jones, 1991) shows it to be a likely route through which nonlemniscal information from the head, face, and mouth would be relayed to the SI.

The 2 compartments of the VPM are clearly delineated in sections stained for $\mathrm{CO}$, which has proven to be a successful marker for many compartments of physiological significance in both the somatic sensory and visual systems (Wong-Riley, 1979; Livingstone and Hubel, 1982, 1983; Horton, 1984; Jones et al., 1986a,b; Wiener et al., 1987). The rodlike organization in the VPM was first described in 1985; the relationships of the rods to the complete contralateral and partial ipsilateral maps of the head, face, and intraoral regions were then demonstrated, and individual rods through the full anteroposterior extent of the VPM were shown to contain the same representation (Jones, 1985; Jones et al., 1986a,b).

In both the ipsi- and contralateral representation, the same small region, for example, the ipsi- or contralateral cheek pouch, is represented through a large anteroposterior extent of the relevant part of the nucleus. When certain of these long anteroposterior domains of common representation were superim- posed on the $\mathrm{CO}$ rod pattern in single animals in our previous studies, it was clear that individual $\mathrm{CO}$ rods could be correlated with a long, anteroposteriorly extended rod of neurons, having receptive fields on the same region of the head, face, or interior of the mouth. The consolidated map showing neurons with the same receptive fields in the same relative position at all anteroposterior levels of the nucleus suggests that this is probably the case for all rods, but multiple recordings from many individual rods will be necessary to confirm this.

From monkey to monkey, there is a certain amount of variation in the number, size, shape, and exact position of $\mathrm{CO}$ rods. Some rods, however, are invariably present. The $2 \mathrm{CO}$ rods at the medial tip of the horizontal part of VPM, which can always be detected, when recorded from invariably represent the ipsilateral side of the hard palate and the ipsilateral pillars of the fauces (see Jones et al., 1986b, their Figs. 6, 7). Two large rods close to the junction between the horizontal and vertical parts of the nucleus are also commonly present and can be identified at approximately the same sites from animal to animal. The mapping results (e.g., Fig. 7) suggest that these would represent the ipsi- and contralateral cheek pouches.

The CO-stained rods have many parallels with similar histochemically distinguished configurations that can be found in VPM of rodents (Killackey and Shinder, 1981; Land and Akhtar, 1987), and which represent the facial vibrissae (Van der 


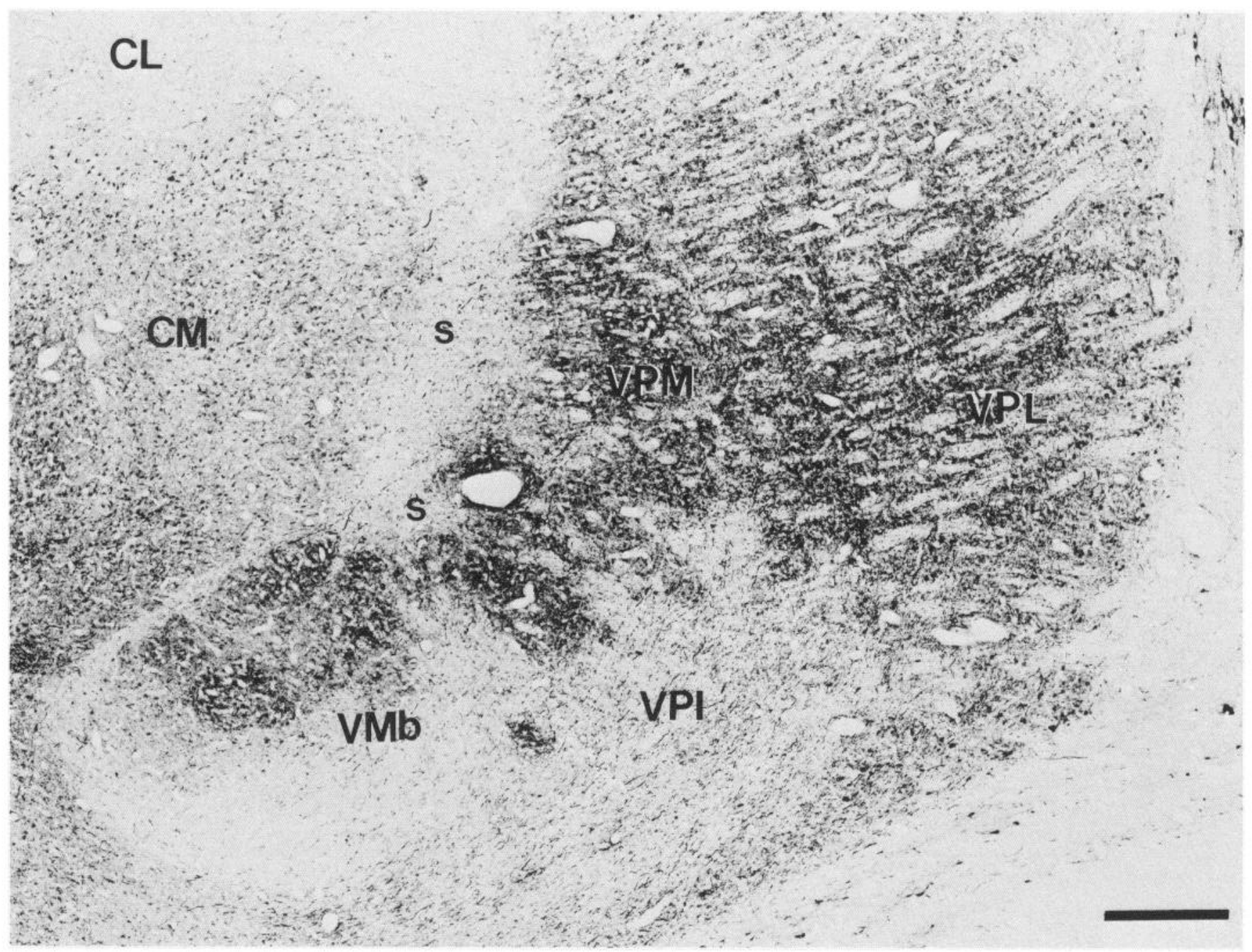

Figure 9. Parvalbumin-immunoreactive section adjacent to Figures $1 A$ and $2 A$. Note the dense staining of the rod domain in the VPM and the reduced or absent staining of cells and neuropil in the small-celled $(s)$ matrix domain and in certain adjacent nuclei. Scale bar, $500 \mu \mathrm{m}$.

Loos, 1976). The rods are also clearly outlined by immunoreactivity for parvalbumin and stained by CAT 301. Elsewhere, it has been reported that the rods stain histochemically for $\mathrm{AChE}$, and that the rods in the contralateral representation stain immunocytochemically for tachykinins (Chalupa et al., 1986; Molinari et al., 1987; Liu et al., 1989). These are further indications of the chemical distinctiveness of the rod compartment. Awareness of the rodlike pattern of organization enables the eye to detect a hint of it even in Nissl stains of adult monkeys (see Fig. 1 and Rausell and Jones, 1991, their Fig. 2), and the rods are especially clear in Nissl stains of the fetal monkey thalamus (Chalupa et al., 1986; Liu et al., 1989).

The CO matrix compartment is a small-celled zone, by contrast with the large- and medium-sized cell population that dominates the rod compartment. The matrix group of cells is distinguished by staining for calbindinlike immunoreactivity. Unlike the rod compartment, it is less distinctive in comparison with the surrounding nuclei, and its cell population seems to have many similarities to the large population of small, calbindin-positive cells also located in relatively weak-staining $\mathrm{CO}$ compartments that form the VPI, VMb, and Pla nuclei and the laminae that separate these from VPM (Jones and Hendry, 1989). In the past, it is likely that the dorsomedial concavity and the ventrolateral expansions of the matrix domain of VPM have been included in these adjacent nuclei by certain authors. Although Olszewski (1952, his Plate XXX) included the smallcelled domain of the dorsomedial concavity in VPM, Jones (1985) included it in Pla or in the lamina separating VPM from CM. It may be the same as the Pom region of Boivie (1979).

Parvalbumin-positive, calbindin-positive, and CAT 301-positive cells in the monkey thalamus are all thalamocortical relay cells (Hendry et al., 1988; Jones and Hendry, 1989), and an important distinction between the rod and matrix compartments of VPM lies in their largely independent innervation by different components of the trigeminal afferent system. The rods are clearly the targets of fibers ascending from the principal trigeminal nucleus. There is a clear-cut segregation of the terminations of fibers ascending with the medial lemniscus from the contralateral nucleus and of those ascending in the dorsal ipsilateral trigeminal tract from the ipsilateral nucleus (Jones et al., 1986b). These terminate in the appropriate representations in VPM and only in the rods. However, where the rod com- 


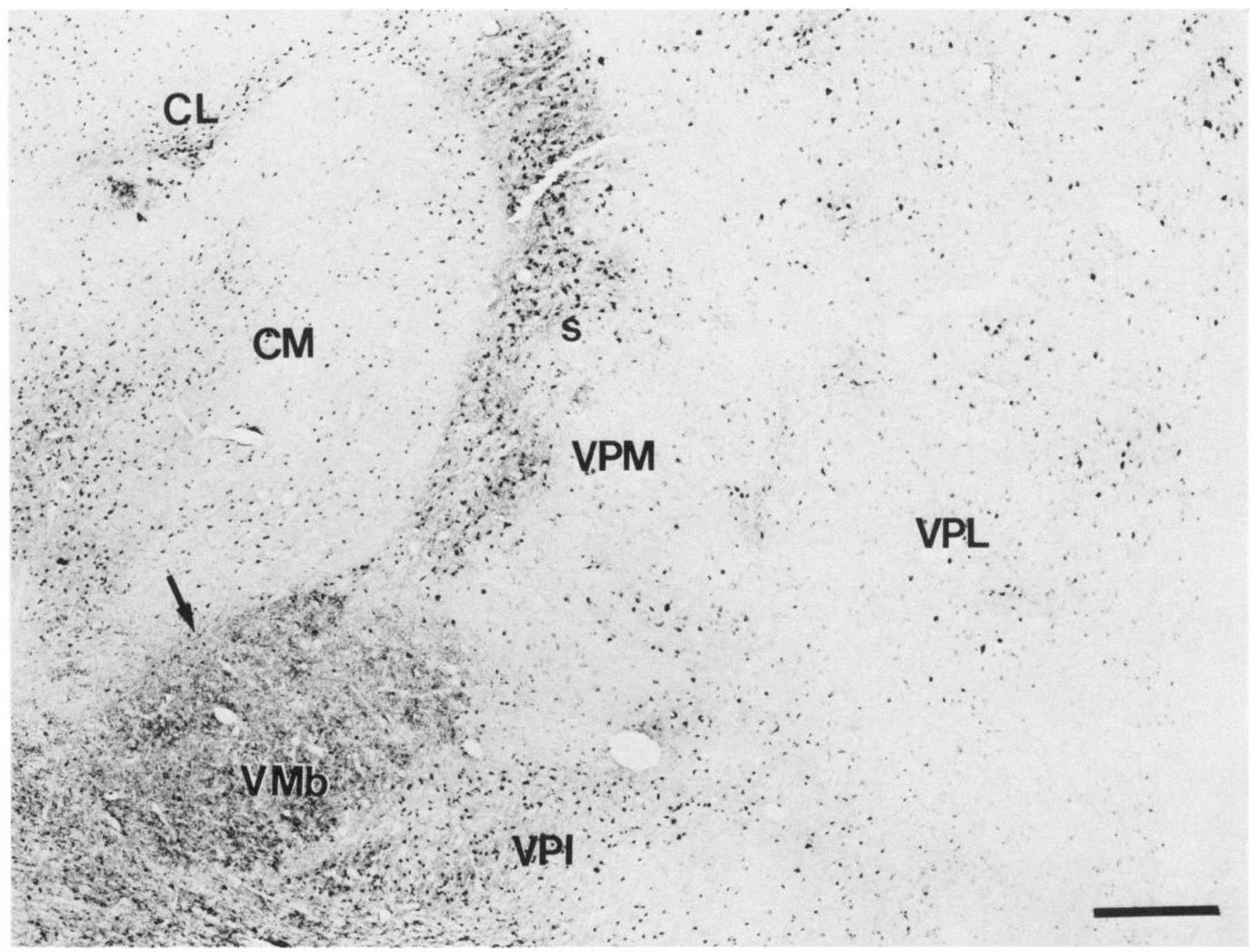

Figure 10. Calbindin-immunoreactive section adjacent to Figures $1 B$ and $2 B$. Note, in comparison with Figure 9 , the increased staining of cells and neuropil in small-celled $(s)$ matrix domain of $V P M$, in most medial rods of $V P M$ (arrow), and in $V M b$ and $V P I$. Note also the islands of stained cells in $V P L$. Scale bar, $500 \mu \mathrm{m}$.

partment appears to be the sole terminus of the pathways from the principal trigeminal nucleus, the matrix compartment is but one component of a much wider field of terminations of fibers ascending from the caudal nucleus of the spinal trigeminal complex (Rausell and Jones, 1991). This wider field includes the central lateral nucleus (CL) and parts of Pla, with scattered ramifications in several other adjacent nuclei such as the posterior nucleus (Po), suprageniculate nucleus (SG), VPI, VLp, and central medial nucleus (CeM). The presence of large populations of calbindin-positive cells in most of these adjacent nuclei (Jones and Hendry, 1989) suggests that calbindin-positive cells may form part of a diffusely organized system of thalamocortical relay cells that, unlike parvalbumin cells, are unconstrained by nuclear borders. The results of the present and our previous single- and multiunit studies give no clue as to the nature of the receptive fields of neurons in the matrix. They may prove to be of a nonlemniscal type, but higher-resolution mapping with finer and much higher-impedance electrodes and more controlled stimuli will be necessary to determine this.

It is clear that the rod compartment of VPM, in particular, has a chemical and molecular identity additional to that conferred by its intense staining for $\mathrm{CO}$. The density of staining for AChE activity and for tachykinin immunoreactivity (Chalupa et al., 1986; Molinari et al., 1987; Liu et al., 1989) has already been mentioned. Approximately $50 \%$ of the relay cells in each rod also stain with the monoclonal antibody CAT 301 (Hendry et al., 1988), which recognizes a surface proteoglycan (Zaremba et al., 1989). The significance of the difference whereby virtually all cortically projecting rod cells are parvalbumin positive (Jones and Hendry, 1988; Rausell and Jones, 1991), but only about half display the surface antigen recognized by CAT 301 , is not clear. It is possible that the CAT 301-positive cells could be selectively innervated by a particular component of the trigeminal lemniscus and project on the SI cortex in some as yet undetermined manner different from that of their companion cells. However, new experiments are needed to determine this. There was nothing in the present study to suggest that CAT 301positive cells are preferentially located in parts of the rods (e.g., the periphery) where they could be contacted by both trigeminal lemniscal and caudal trigeminal fibers. A previous study (Hen- 


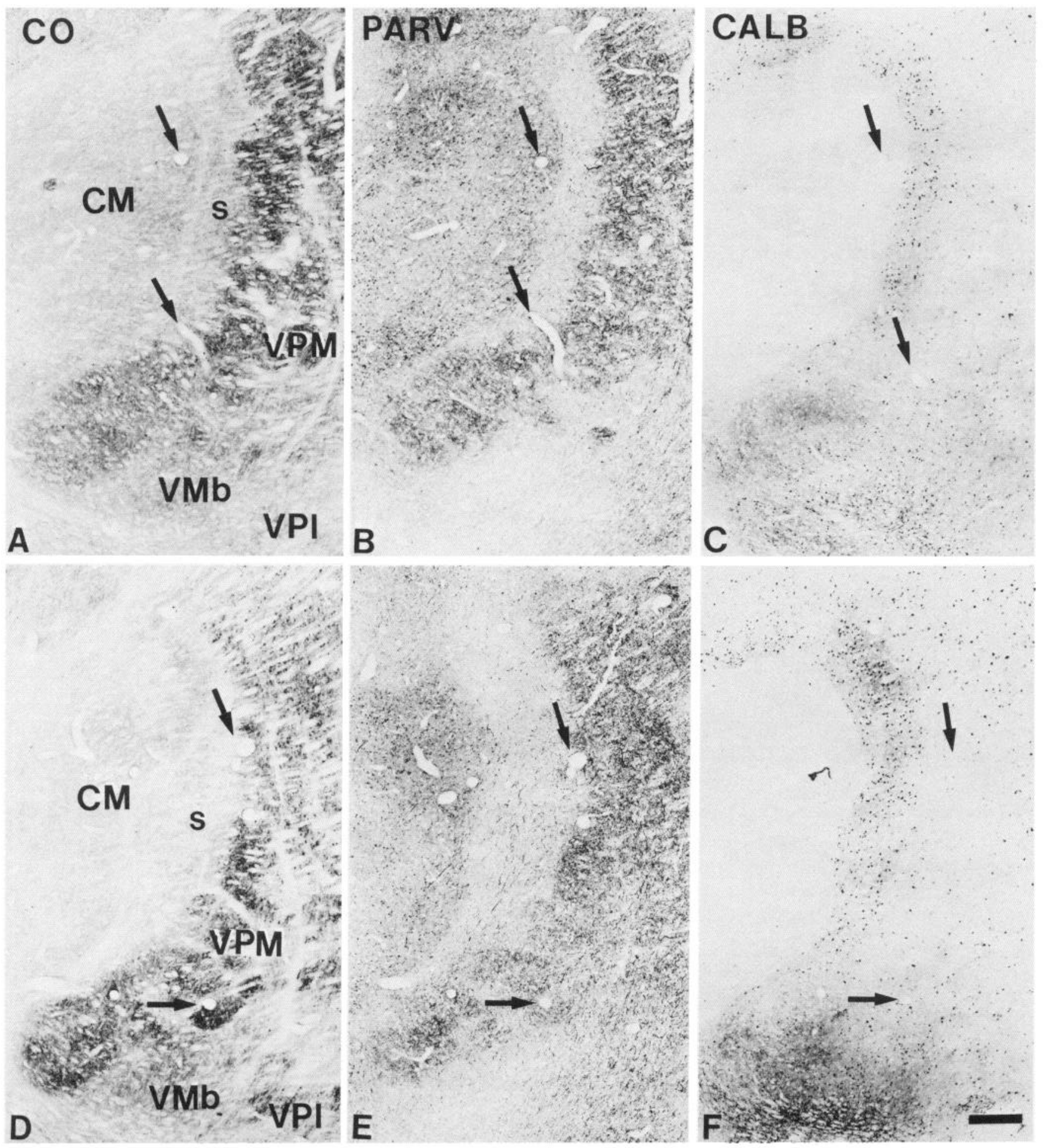

Figure 11. Each trio $(A-C, D-F)$ of sections from series of same monkey are immediately adjacent to one another and stained for CO (A, $D)$, parvalbumin $(P A R V ; B, E)$, or calbindin $(C A L B ; C, F)$. The same blood vessels are arrowed in $A-C$ and in $D-F$. Note the similarity in CO and parvalbumin staining and the coincidence of $\mathrm{CO}$ rods and parvalbumin rods (e.g., close to the lower arrow in $D, E$.). Note also the complementary nature of calbindin staining. Scale bar, $500 \mu \mathrm{m}$. 


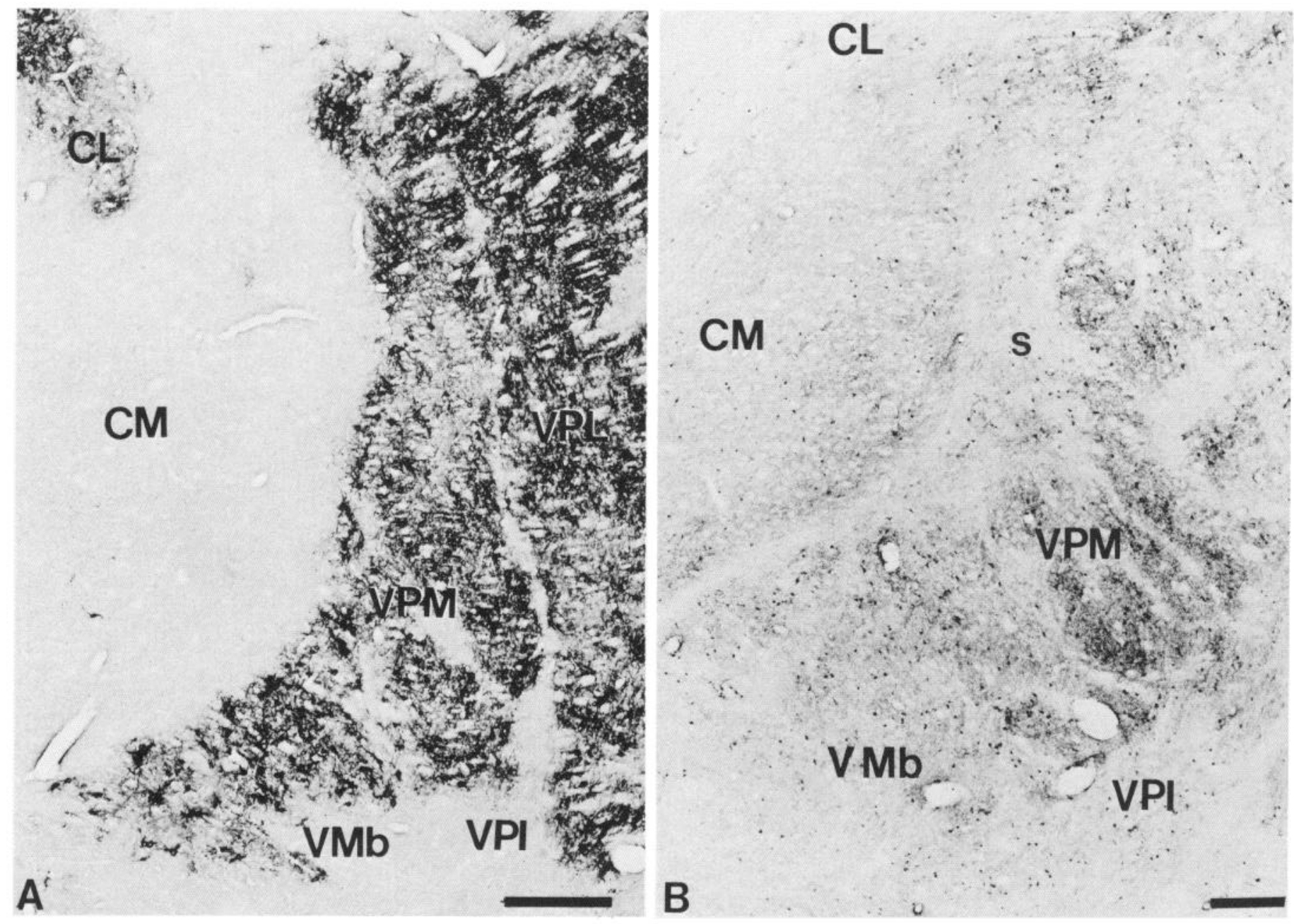

Figure 12. A, CAT 301-stained section showing staining of cells and neuropil in regions corresponding to the rods of VPM, but absence of staining in complementary regions corresponding to the matrix. Scale bar, $500 \mu \mathrm{m}$. B, Section at a similar level from a different monkey, stained immunocytochemically for GABA and showing increased density of cell and neuropil staining in regions corresponding to the rods but with reduction in regions corresponding to the small-celled $(s)$ matrix domain. Scale bar, $250 \mu \mathrm{m}$.

dry et al., 1988) indicated that clusters of CAT 301-positive cells tend to interdigitate with the major densities of trigeminal lemniscal fiber terminations in each rod.

The GABA-immunoreactive interneurons of VPM also show a preferential concentration in the rods in comparison with the matrix compartment, where far fewer are found. The rod compartment also shows a far higher density of GABAimmunoreactive, terminal-like puncta than the matrix compartment. Relay cells in both compartments thus appear subject to GABA-mediated inhibition. The focal concentration in the rods, however, would tend to suggest that each rod is a selfcontained unit in terms of these inhibitory phenomena, whereas the appearance of the matrix suggests a more diffuse, perhaps less specific organization. The concept of the thalamic rod as an essentially self-contained unit is supported by the results of the previous studies, which show the physiologically defined rod to be a place- and modality-specific entity whose anatomical equivalent projects to a focal domain in the SI cortex (Jones and Friedman, 1982; Jones et al., 1982). The projection of the rods to focal domains in the SI cortex probably forms an essential basis for the columnlike organization of SI first demonstrated by Mountcastle (1957) and Powell and Mountcastle (1959). The focal nature of the GABA cell distribution probably ensures that "cross-talk" between neighboring rods and between inputs to different cortical columns is restricted, as well as providing all the machinery necessary for local inhibitory phenomena operating at the single-cell level within the rod. 


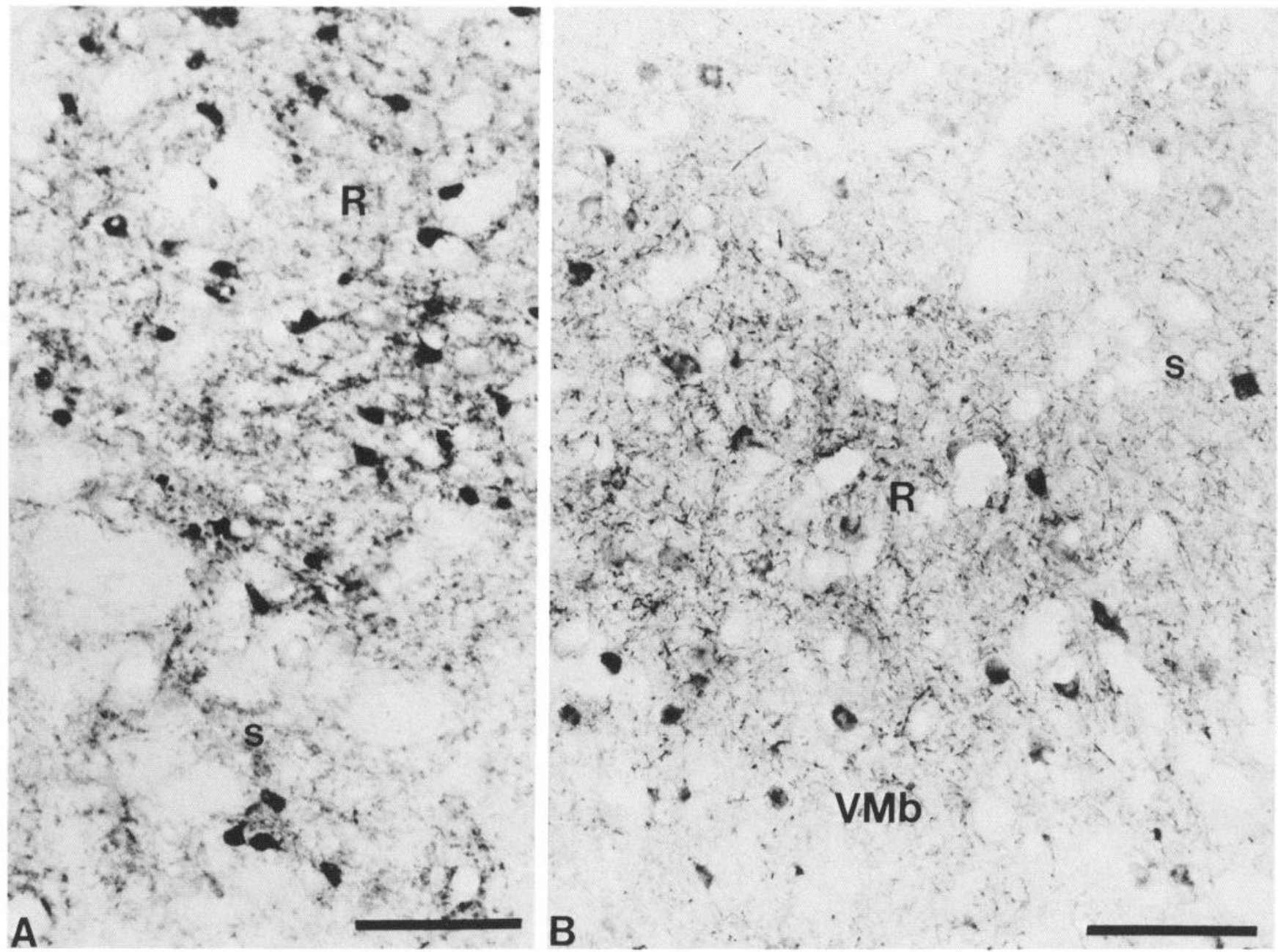

Figure 13. A, High magnification of the rod $(R)$ and matrix $(s)$ domains, showing differential density of cell and neuropil immunoreactivity for GABA (cf. Fig. 11). B, Enhanced calbindin immunoreactivity in one of the medial rods $(R)$ of $V P M$. Scale bars, $100 \mu \mathrm{m}$.

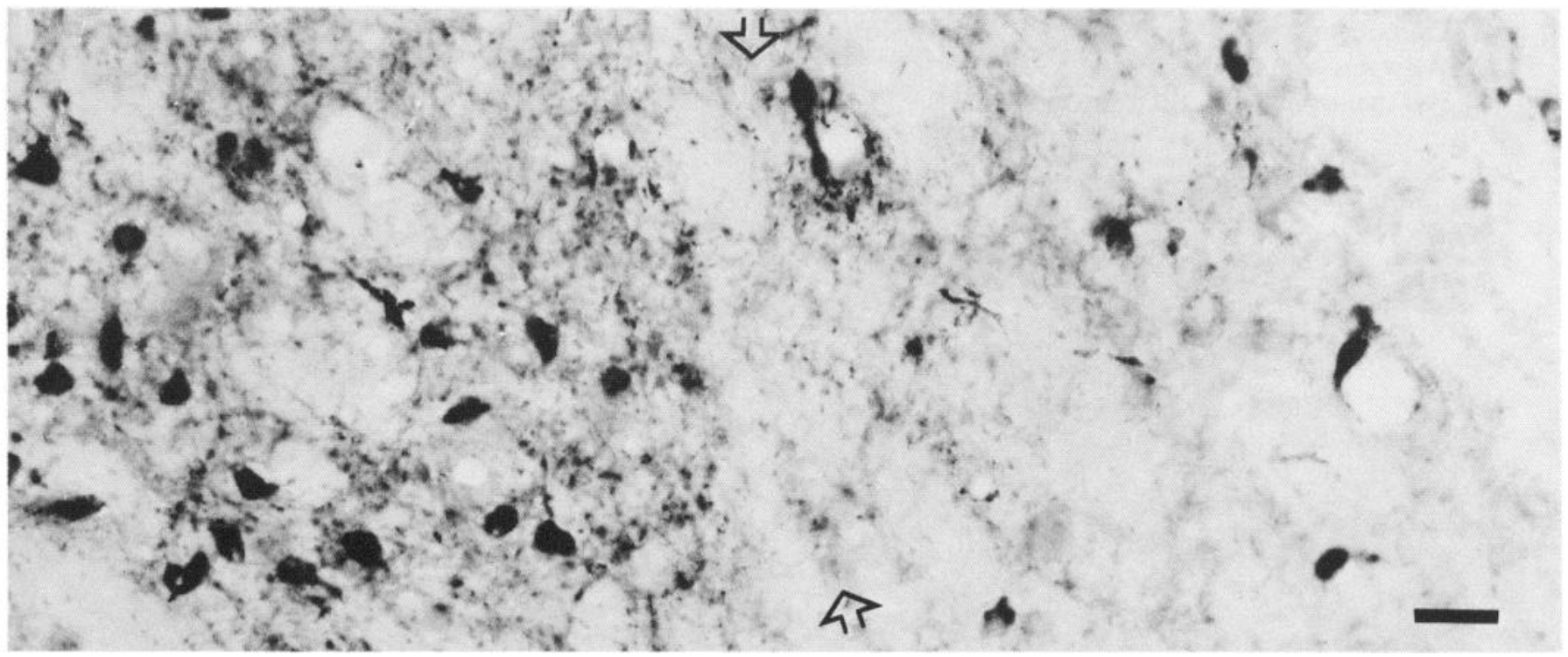

Figure 14. Higher magnification of part of a GABA-immunoreactive section, showing border (arrows) between parts of rod (left) and matrix (right) domains. Note the larger number of GABA-immunoreactive cells and especially of stained puncta in the rod domain. Scale bar, $50 \mu \mathrm{m}$. 


\section{References}

Boivie J (1979) An anatomical reinvestigation of the termination of the spinothalamic tract in the monkey. J Comp Neurol 186:343-370.

Chalupa LM, Hendry SHC, Jones EG, Killackey HP, Molinari M (1986) Localized substance $P$ immunoreactivity in the VPM thalamic nucleus of adult and fetal monkeys. Soc Neurosci Abstr 12:328.

Dykes RW (1983) Parallel processing of somatosensory information: a theory. Brain Res Rev 6:47-1 15.

Dykes RW, Sur M, Merzenich MM, Kaas JH, Nelson RJ (1981) Regional segregation of neurons responding to quickly adapting, slowly adapting, deep and pacinian receptors within thalamic ventroposterior lateral and ventroposterior inferior nuclei in the squirrel monkey (Saimiri sciureus). Neuroscience 6:1687-1692.

Florence SL, Wall JT, Kaas JH (1988) The somatotopic pattern of afferent projections from the digits to the spinal cord and cuneate nucleus in macaque monkeys. Brain Res 452:388-392.

Hendry SHC, Hockfield S, Jones EG, McKay R (1984) Monoclonal antibody that identifies subsets of neurons in the central visual system of monkey and cat. Nature 307:267-269.

Hendry SHC, Jones EG, Hockfield S, McKay RDG (1988) Neuronal populations stained with the monoclonal antibody CAT-301 in the mammalian cerebral cortex and thalamus. J Neurosci 8:518-542.

Hockfield S, McKay RDG (1983) A surface antigen expressed by a subset of neurons in the vertebrate central nervous system. Proc Natl Acad Sci USA 80:5758-5761.

Horton JC (1984) Cytochrome oxidase patches: a new cytoarchitectonic feature of monkey visual cortex. Philos Trans R Soc Lond Biol 304:199-253.

Jones EG (1985) The thalamus, pp 255-256. New York: Plenum.

Jones EG, Friedman DP (1982) Projection pattern of functional components of thalamic ventrobasal complex on monkey somatosensory cortex. J Neurophysiol 48:521-544.

Jones EG, Hendry SHC (1989) Differential calcium binding protein immunoreactivity distinguishes classes of relay neurons in monkey thalamic nuclei. Eur J Neurosci 1:222-246.

Jones EG, Friedman DP, Hendry SHC (1982) Thalamic basis of place and modality-specific columns in monkey somatosensory cortex: a correlative anatomical and physiological study. J Neurophysiol 48: 545-568.

Jones EG, Hendry SHC, Brandon C (1986a) Cytochrome oxidase staining reveals functional organization of monkey somatosensory thalamus. Exp Brain Res 62:438-442.

Jones EG, Schwark HD, Callahan PA (1986b) Extent of the ipsilateral representation in the ventral posterior medial nucleus of the monkcy thalamus. Exp Brain Res 63:310-320.

Kaas JH, Nelson RJ, Sur M, Dykes RW, Merzenich MM (1984) The somatolopic organization of the ventroposterior thalamus of the squirrel monkey, Saimiri sciureus. J Comp Neurol 226:1 11-140.

Killackey H, Shinder A (1981) Central correlates of peripheral pattern alterations in the trigeminal system of the rat. II. The effect of nerve section. Dev Brain Res 1:121-126.

Land PW, Akhtar ND (1987) Chronic sensory deprivation affects cytochrome oxidase staining and glutamic acid decarboxylase im- munoreactivity in adult rat ventrobasal thalamus. Brain Res 425: $178-181$.

Liu X-B, Jones EG, Huntley GW, Molinari M (1989) Tachykinin immunoreactivity in terminals of trigeminal afferent fibers in adult and fetal monkey thalamus. Exp Brain Res 78:479-488.

Livingstone MS, Hubel DH (1982) Thalamic inputs to cytochrome oxidase-rich regions in monkey visual cortex. Proc Natl Acad Sci USA 779:6098-6106.

Livingstone MS, Hubel DH (1983) Specificity of corticocortical connections in monkey visual system. Nature 304:532-534.

Matute C, Streit P (1986) Monoclonal antibodies demonstrating GABAlike immunoreactivity. Histochemistry 56:147-157.

McKay RDG, Hockfield SJ (1982) Monoclonal antibodies distinguish antigenically discrete neuronal types in the vertebrate central nervous sytem. Proc Natl Acad Sci USA 79:6747-6751.

Molinari M, Hendry SHC, Jones EG (1987) Distributions of certain neuropeptides in the primate thalamus. Brain Res 426:270-289.

Mountcastle VB (1957) Modality and topographic properties of single neurons of cat's somatic sensory cortex. J Neurophysiol 20:408-434.

Mountcastle VB (1984) Central nervous mechanisms in mechanoreceptive sensibility. In: Handbook of physiology: the nervous system II (Darian-Smith I ed), pp. 789-897. Washington, DC: American Physiological Society.

Olzsewski J (1952) The thalamus of the Macaca mulatta. An atlas for use with the stereotaxic instrument. Basel: Karger.

Powell TPS, Mountcastle VB (1959) Some aspects of the functional organization of the cortex of the postcentral gyrus of the monkey: a correlation of findings obtained in a single unit analysis with cytoarchitecture. Bull Johns Hopkins Hosp 105:133-162.

Rausell E, Jones EG (1989) Modular organization of the thalamic VPM nucleus in monkeys. Soc. Neurosci Abstr 15:311.

Rausell E, Jones EG (1991) Chemically distinct compartments of the thalamic VPM nucleus in monkeys relay principal and spinal trigeminal pathways to different layers of the somatosensory cortex. J Neurosci 11:226-237.

Van der Loos H (1976) Barreloids in mouse somatosensory thalamus. Neurosci Lett 2:1-6.

Werner G, Whitsel BL (1968) Topology of the body representation in somatosensory area I of primates. J Neurophysiol 31:856-869.

Wiener SI, Johnson JI, OstapoffEM (1987) Demarcations of the mechanosensory projection zones in the raccoon thalamus, shown by cytochrome oxidase, acetylcholinesterase, and Nissl stains. J Comp Neurol 258:509-526.

Wong-Riley M (1979) Changes in the visual system of monocularly sutured or enucleated cats demonstrable with cytochrome oxidase histochemistry. Brain Res 171:11-28.

Woolf NJ, Butcher LL (1981) Cholinergic neurons in the caudateputamen complex proper are intrinsically organized: a combined Evans blue and acetylcholinesterase analysis. Brain Res Bull 7:487-508.

Zaremba S, Guimaraes A, Kalb RG, Hockfield S (1989) Characterization of an activity-dependent, neuronal surface proteoglycan identified with monoclonal antibody CAT-301. Neuron 2:1207-1219. 